DIFFUSION OF INNOVATIONS IN HEALTH SERVICE ORGANISATIONS: A SYSTEMATIC LITERATURE REVIEW. Greenhalgh T, Robert G, Bate P, Macfarlane F, Kyriakidou O. Oxford: Wiley-Blackwell; 2005. 328 p.

ISBN-13: 978-0727918697

A inovação tornou-se tema de interesse crescente de gestores, profissionais e pesquisadores da área da saúde no Brasil, nos últimos anos 1,2,3. À proporção que cresce esse interesse, mais e mais se percebe a extensão em que as iniciativas de inovação podem favorecer mudanças nas formas de organização e gestão da saúde.

Um aspecto-chave das iniciativas de inovação em saúde, porém, tem sido relativamente desprezado no presente interesse pelo tema: o que se refere à difusão de inovações nas organizações prestadoras de serviços. Trata-se da disseminação planejada de novas rotinas e maneiras de trabalhar, com o fim de melhorar a efetividade do atendimento, reduzir custos e aumentar a eficiência das organizações. É a difusão assim orientada que permite maximizar a exposição e o alcance de inovações bem sucedidas no âmbito dos comportamentos e, eventualmente, induzir mudanças desejáveis não só nas organizações prestadoras de serviços, mas também na prática clínica.

O livro Diffusion of Innovations in Health Service Organisations: A Systematic literature Review, de um grupo de pesquisadores do University College, Londres, e da University of Surrey, Guilford, ambas no Reino Unido, faz uma extensa revisão da literatura relevante de pesquisa sobre difusão de inovações nas organizações prestadoras de serviços de saúde. Embora tenha sido escrito para atender a uma demanda da Agência de Modernização do Serviço Nacional de Saúde da GrãBretanha, o livro constitui uma referência indispensável, e ainda atual, para estudiosos da difusão de inovações em saúde em qualquer país onde o tema desperte interesse.

A difusão de inovações abrange genericamente uma gama de atividades relativas à propagação de novos produtos, tecnologias, processos, idéias, comportamentos etc., em contextos sociais e mercadológicos 4,5,6. O tema ganhou impulso no Brasil em meados da década de 1990, quando as iniciativas de inovação derivadas dos convênios, intercâmbios e parcerias que então se desenvolviam entre empresas, governos, universidades, incubadoras e instituições de pesquisa começaram a requerer maior circulação de informações e de conhecimento. Esse desdobramento permitiu estender o foco do interesse pelas inovações, até então centrado nas relações estritamente econômicas entre mercado e indústria, para variáveis de natureza social e comportamental.

A maioria das pesquisas sobre difusão de inovações visa a identificar os fatores que influenciam a adoção ou não de uma inovação. Os estudos nessa área remontam ao fim do século XIX, quando o psicólogo social francês Jean-Gabriel Tarde (1843-1904) formulou um conjunto de princípios gerais que realçavam o papel da difusão nos processos de mudança social. Quase concomitantemente, antropólogos europeus analisaram a mudança social em termos da difusão de inovações. Foi, porém, uma pesquisa empírica sobre a difusão de um tipo de milho híbrido entre fazendeiros do estado norte-americano de Iowa, na década de 1940 7, que deu impulso à área. Desde então, da sociologia rural ao marketing, os estudos sobre difusão de inovações vêm se realizando com diferentes abordagens, conceitos e métodos numa variedade de disciplinas e áreas de conhecimento, entre as quais a saúde pública, geografia, sociologia, antropologia, comunicação, economia, ciências administrativas, educação e psicologia.

Uma referência teórica freqüente em grande parte dos estudos da área tem sido o modelo de difusão de inovações desenvolvido pelo comunicólogo norteamericano Everett Rogers (1931-2004). O livro de Greenhalgh et al. faz uso da sistematização mais acabada desse modelo 6 para mapear e examinar criticamente cerca de mil publicações relativas à propagação e à manutenção de inovações nas organizações de saúde indo além, às vezes, deste intento, quando os autores recorrem a uma literatura menos especializada para enriquecer o texto com considerações suplementares de natureza conceitual, empírica, teórica e metodológica (a obra de Rogers, originalmente publicada em 1962, foi sucessivamente atualizada em quatro novas edições, a última em 2003).

Dividido em 11 capítulos e quatro apêndices, o livro organiza metodicamente os dados e informações cientificamente significativos de uma literatura de pesquisa tão vasta que, segundo Rogers 6 , estava perto de atingir a marca de quatro mil publicações num período de 23 anos. As questões mais relevantes para os serviços de saúde são agrupadas em seis tópicos de caráter geral: (a) a inovação propriamente dita; (b) o processo de adoção de inovações; (c) a difusão e a disseminação (a primeira informal, a segunda formal e planejada, conforme a definição dos autores); (d) o contexto interno das organizações de saúde; (e) o contexto interorganizacional externo; e (f) o processo de implantação e manutenção das inovações.

O livro cobre uma variedade de assuntos, incluindo as diversas tradições de pesquisa na área, as relações entre os chamados atributos das inovações e a efetiva adoção destas inovações (com uma pertinente discussão sobre questões epistemológicas relativas ao conceito de atributo de inovação), os estudos que relacionam a difusão nas organizações prestadoras de serviços de saúde a características pessoais dos adotantes, o papel dos líderes de opinião e das redes interpessoais na difusão, e as influências organizacionais e ambientais na inovação.

Como produto desse meticuloso esforço de revisão sistemática, os autores acabam por desenvolver um modelo conceitual próprio de difusão de inovações, 
que é descrito e aplicado em quatro estudos de caso de difusão em organizações de saúde, nos capítulos finais. Recomendações práticas acompanham a descrição do modelo, tendo em vista futuras adaptações. O leitor pode ainda se beneficiar de sugestões de pesquisa desenvolvidas ao longo do trabalho e, nos apêndices, de um detalhamento adicional dos estudos empíricos pesquisados, que são listados e descritos sistematicamente numa variedade de categorias de revisão bibliográfica, entre as quais se incluem resumos dos procedimentos de coleta de dados, sínteses dos resultados principais e comentários dos autores.

A leitura de Diffusion of Innovations in Health Service Organisations: A Systematic Literature Review oferece elementos para uma avaliação acurada da importância da difusão de inovações na implantação de melhores práticas nas organizações prestadoras de serviços de saúde. Em que pese essa importância, é preciso sublinhar que a literatura de pesquisa sobre difusão de inovações tem sido alvo de críticas de pesquisadores comportamentais a flagrantes debilidades teóricas, conceituais e metodológicas encontradas nos modelos dominantes 8,9,10. A insuficiente densidade teórica, a relativa despreocupação dos pesquisadores da área para com a operacionalização dos conceitos, e a precária metodologia de coleta de dados (caracteristicamente baseada em levantamentos verbais, post-hoc, de lembranças pessoais das circunstâncias da adoção) são três aspectos importantes dessas críticas. Os autores do livro estão conscientes de tais limitações, embora passem ao largo de uma abordagem propriamente comportamental e metodologicamente mais rigorosa.

Seja como for, as publicações revisadas pelos autores são representativas do estado da arte da pesquisa na área, o que torna a obra uma referência única no seu gênero para gestores, profissionais e pesquisadores com interesse no tema da inovação na área da saúde.

João Bosco Jardim

Centro de Pesquisa René Rachou, Fundação Oswaldo Cruz, Belo Horizonte, Brasil.

jardim@cpqrr.fiocruz.br

1. Buss PM. Inovação tecnológica em saúde na Fundação Oswaldo Cruz. Hist Cienc Saude-Manguinhos 2003; 10 Suppl 2:836-42.

2. Caetano R, Vianna CDM. Processo de inovação tecnológica em saúde: uma análise a partir da organização industrial. Cad Saúde Colet (Rio J) 2006; 14:95-112.

3. Gadelha CAG. O complexo industrial da saúde e a necessidade de um enfoque dinâmico na economia da saúde. Ciênc Saúde Coletiva 2003; 8:521-35.

4. Bandura A. Social foundations of thought and action: A social cognitive theory. Englewood Cliffs: Prentice Hall; 1986.

5. Oldenburg B, Parcel GS. Diffusion of innovations. In: Glanz K, Rimer BK, Lewis FM, editors. Human behavior and health education: theory, research and practice. $3^{\text {rd }}$ Ed. San Francisco: Jossey-Bass; 2002. p. 312-34.

6. Rogers EM. Diffusion of innovations. 4th Ed. New York: Free Press; 1995.

7. Ryan B, Gross NC. The diffusion of hybrid seed in two Iowa communities. Rural Sociol 1943; 8:15-24.
8. Pennypacker HS. The challenge of technology transfer: buying it without selling out. Behav Anal 1986; 9:147-56.

9. Stolz SB. Adoption of innovations from applied behavioral research: does anybody care? J Appl Behav Anal 1981; 14:507-11.

10. Winett RA. Information and behavior: systems of influence. Hillsdale: Erlbaum; 1986.

\section{ASSISTÊNCIA FARMACÊUTICA E ACESSO A} MEDICAMENTOS. Oliveira MA, Bermudez JAZ, Osório-de-Castro CGS. Rio de Janeiro, Editora Fiocruz; 2007. 112 p.

ISBN: 978-85-7541-131-5

Os autores do livro Assistência Farmacêutica e Acesso a Medicamentos recuperam fatos históricos, legislação, questões éticas e de direitos humanos que ajudam a compreender os determinantes econômicos e políticos para assegurar a eqüidade, o direito à saúde e o acesso aos medicamentos, bem como o incremento e a qualificação das ações envolvidas na assistência farmacêutica.

O livro está estruturado em quatro capítulos e aborda de forma clara e objetiva o conceito e definição de assistência farmacêutica, seja na prática gerencial no provimento de medicamentos (acesso), seja na prática clínica (cuidados farmacêuticos) em todas as etapas constitutivas do ciclo de assistência farmacêutica. As atividades desenvolvidas na assistência farmacêutica, segundo a Política Nacional de Medicamentos 1, têm como objetivo precípuo garantir o acesso da população a medicamentos essenciais de qualidade, promovendo, dessa forma, seu uso racional. A obra também traz importante contribuição para o debate sobre a inexistência, fragilidade, inconsistência e insuficiência de aplicação de recursos e investimentos na pesquisa e desenvolvimento de inovações terapêuticas e na implementação de programas de saúde voltados ao atendimento das necessidades dos países em desenvolvimento, o que atravanca o acesso a medicamentos de grandes contingentes populacionais que mais precisam de tais insumos em saúde. Além disso, os autores discorrem sobre o sistema internacional de propriedade intelectual e suas implicações no acesso a medicamentos essenciais.

No primeiro capítulo, Assistência Farmacêutica: Campo Conceitual e de Prática, a definição de assistência farmacêutica proposta pela Organização Mundial da Saúde (OMS) - "assistência farmacêutica como um grupo de serviços e atividades relacionados com o medicamento, destinados a apoiar as ações da saúde que demanda a comunidade, os quais devem ser efetivados através da entrega expedita e oportuna dos medicamentos a pacientes hospitalizados e ambulatoriais, garantindo os critérios de qualidade na farmacoterapia" (p. 14) - é utilizada pelos autores para discorrer sobre os conceitos e diretrizes referentes à implementação das atividades relativas à assistência farmacêutica no Brasil, respaldada pela homologação da Política Nacional de Medicamentos.

Os autores referem que a assistência farmacêutica é um processo dinâmico e multidisciplinar, que visa a abastecer os sistemas, programas ou serviços de saúde com medicamentos de qualidade, viabilizando, consequentemente, o acesso de pacientes a medicamentos 